\title{
Collaboration through Concept Maps
}

\author{
Brian R Gaines and Mildred L G Shaw \\ Knowledge Science Institute, University of Calgary
}

\begin{abstract}
Concept maps have been used in education, policy studies and the philosophy of science to provide a visual representation of knowledge structures and argument forms. They provide a complementary alternative to natural language as a means of communicating knowledge. In many disciplines various forms of concept map are already used as formal knowledge representation systems, for example: semantic networks in artificial intelligence, bond graphs in mechanical and electrical engineering, Petri nets in communications, and category graphs in mathematics. This paper describes the design and applications of groupware concept mapping tools designed to support collaboration in dispersed learning communities.
\end{abstract}

Keywords - Concept maps, World-Wide Web, collaborative learning

\section{Introduction}

It is no coincidence that the words image and imagination have the same roots. It has been known from early times that visual imagery plays a significant role in the creative processes of many people (Arnheim, 1969). The availability of low-cost, highperformance graphic workstations and personal computers has made it possible to support visual thinking processes in a variety of ways across a wide range of disciplines, and again has led to a major literature on visualization (Nielson and Rosenblum, 1991). There is also a large and growing literature on the use of computer-based tools to support visual languages for a wide variety of structures (Glinert, 1990).

The activities reported in this article are part of a long-term program of research on the technology and applications of computer-based collaborative systems to support individuals and groups in creative visualization. This report focuses on our studies of concept mapping techniques, their implementation on personal computers, their use in collaborative learning over digital networks, and their integration with WorldWide Web (WWW) servers and browsers.

\section{Concept Maps}

Many disciplines developed visual languages for 'concept maps', 'cognitive maps' and 'argument forms.' In education, Novak and Gowin (Novak, 1977) developed a system of concept maps that has been widely applied in the evaluation of students' learning in the school system (Novak and Gowin, 1984)—Figure 1 shows a map from these studies.

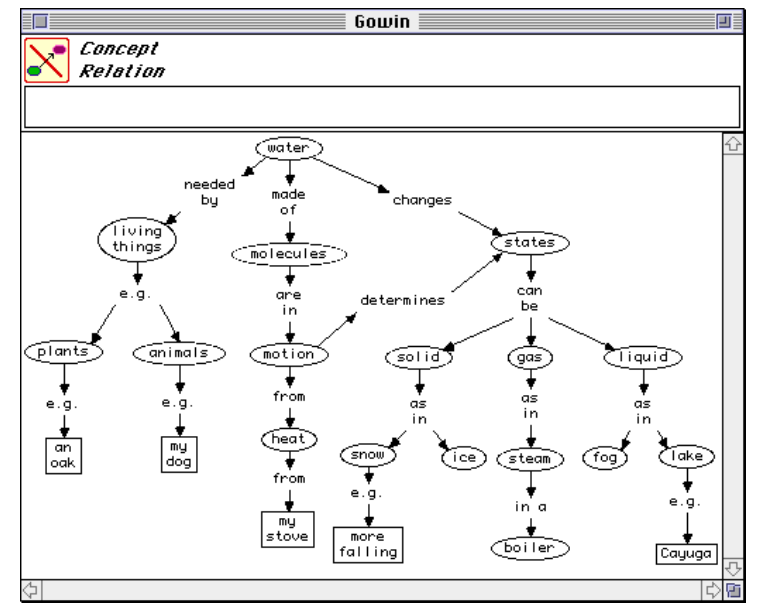

Figure 1 Concept map of student's knowledge

A wide variety of different forms of concept map have been applied in education (Lambiotte, Dansereau, Cross and Reynolds, 1989). In management, Axelrod proposed cognitive maps as a means of representing the conceptual structures underlying decision making (Axelrod, 1976). In the history of science, the dynamics of concept maps have been used to represent the processes of conceptual change in scientific revolutions (Nersessian, 1989; Thadgard, 1992). In the philosophy of science, Toulmin (1958) developed a theory of scientific argument based on typed concept maps.

\section{KMap: A Concept-Mapping Tool}

We have developed a general concept mapping tool called KMap (Gaines and Shaw, 1993; Gaines and Shaw, 1994) which provides a grapher for nodes and arcs that can be programmed by the user to support different forms of concept map. User interaction with KMap takes place through the creation of statements in the visual language, and through interaction with such 
statements through popup menus whose content is specific to node type. The action initiated is contextsensitive: to the node selected for the popup, to nodes linked to it, and to other nodes preselected by clicking on them. This allows complex activities to be initiated by natural user actions.

Figure 1 was drawn in KMap, and Figure 2 shows one of local applications (Gaines and Shaw, 1993) in graduate education where students are introduced to the structure of a research program through an interactive concept map that may be edited and linked to their own research. The node types and content may be edited by the students, as may a database of information attached to the nodes that provides links to other concept maps and files, either locally or across the Internet. For example, the student has moused over a node on objectives concerned with the determination of the current state of the art, and moused down when the cursor changed to a popup menu icon to access a menu giving access to a local file of her supervisor's notes and a WWW file of her own notes put up as part of a course.

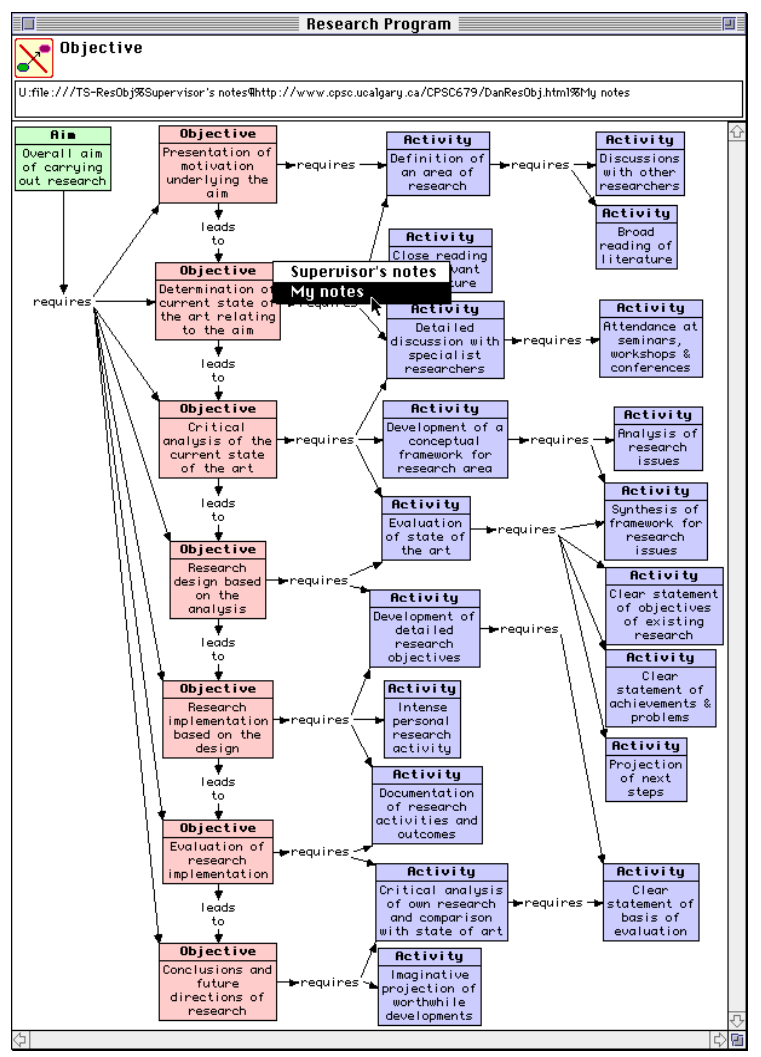

Figure 2 Concept map on graduate research

KMap is programmable through the Apple highlevel object event protocol, and can be scripted by any of the languages in Apple's open scripting architecture. It can also run scripts triggered by user interaction. The combination of these capabilities enables KMap to be integrated with other applications, and user interaction with graphical structures in the visual language to be used to control any activity supported on the host computer or network.

Figure 3 shows a user interacting with a map of multimedia materials concerned with the identification of birds. The map gives an overview of the topic and acts as an index to available material. As the user mouses over a node a popup menu symbol appears. Clicking on this displays a menu that can be used to access the material, display text, play a sound, run a movie, and so on. The menu is generated from an underlying script, and user actions are reported to the script. The material accessed can be retrieved using either standard facilities for sound and movie playing within the application, or through commands which initiate another application and appropriate dataset. The actions can include the opening of other concept maps so that is possible to index a large body of material through layers of connected maps.

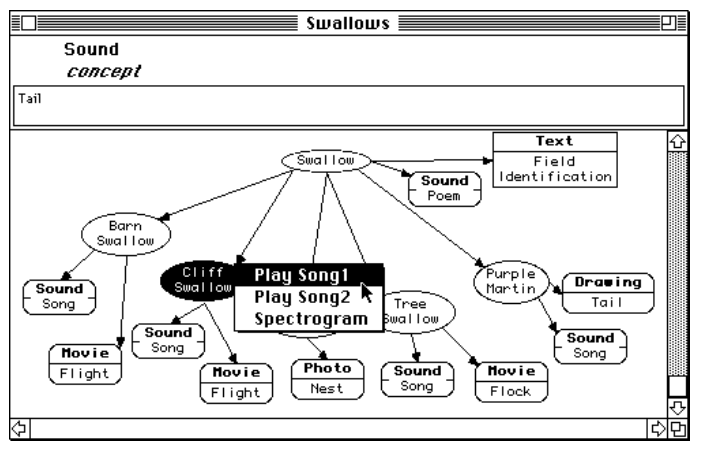

Figure 3 Concept map of multimedia materials

Figure 4 shows the generation of the visual language used in Figure 3. The designer generates nodes of different types appropriate to the domain of materials to be accessed. The visual features of each type can be designed to be distinctive and attractive. The script can associate simple retrieval activities for each type dependent on the data stored with each node, or, since it has full access to the concept map, can take context-sensitive actions as appropriate.

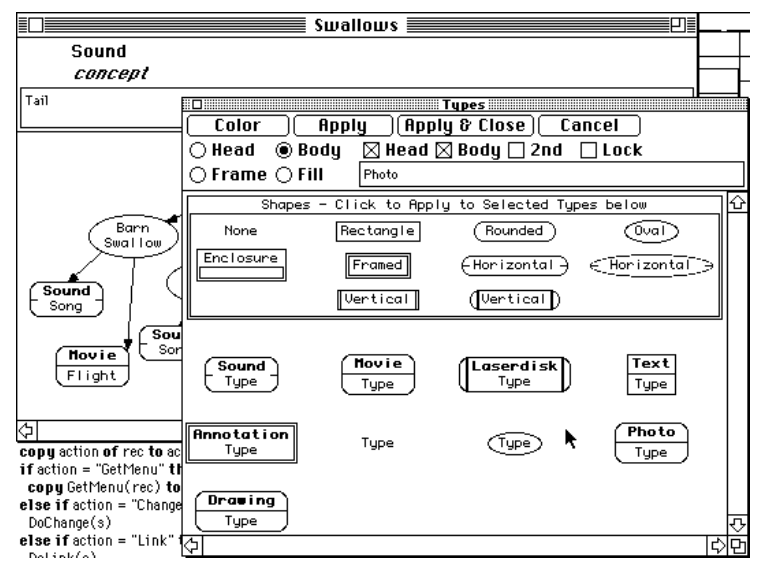

Figure 4 Entering concept map types to define a visual language 


\section{Collaborative Access to Concept Maps}

One of the major objectives of our research in the last two years has been to port all our interactive systems, currently on personal computers and local area networks, to operate effectively in the open, wide area networking of the Internet, and, wherever possible, to integrate seamlessly with World-Wide Web. However, the graphic primitives necessary for interactive concept maps are not provided in HTML and it is a challenge to port concept maps to WWW.

It proved simple to operate KMap as a WWW client helper, capable of accepting concept maps brought across the WWW by a browser such as Netscape, and of requesting arbitrary files, including concept maps, to be fetched through messages sent from KMap scripts to the WWW browser. Figure 5 shows an HTML document in Netscape which has links to KMap documents.

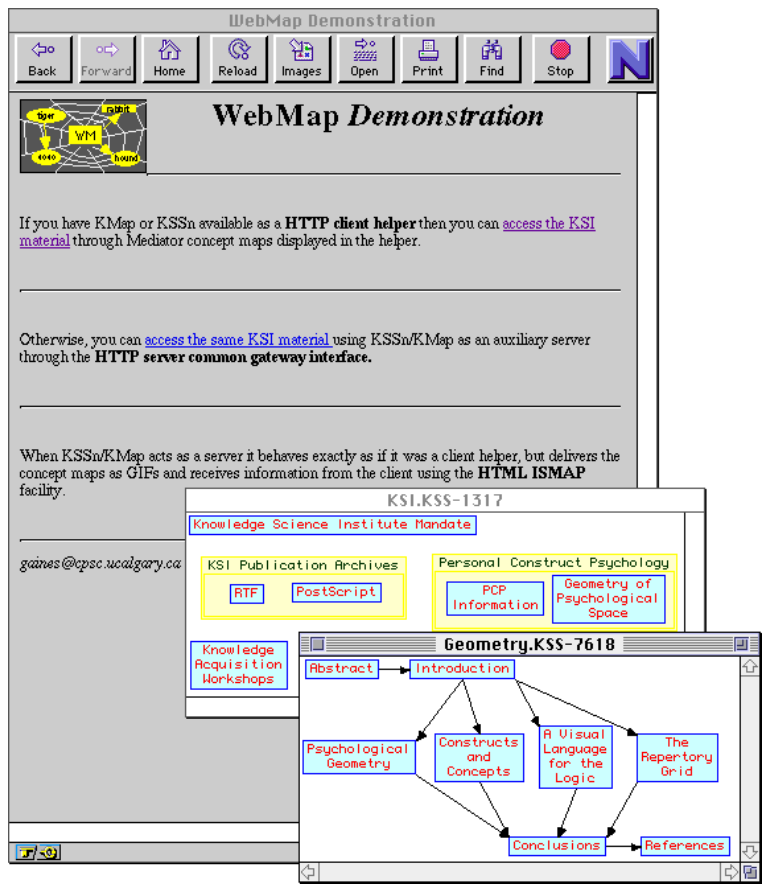

Figure 5 KMap acts a client helper application to the NetScape browser

When the user clicks on the hypertext link "access the KSI material" at the top of the screen, Netscape fetches a KMap file "KSI.KSS" which it passes to KMap which open and displays it as shown in the KMap window at the middle right.

Clicking on the node "Geometry of Psychological Space" at the right of this map causes KMap to send a message to Netscape requesting that the file with url "http://ksi.cpsc.ucalgary.ca/WebMap/Geometry.KSS" be fetched. This is a concept map of the structure of an article that opens in another KMap window shown at bottom right. Clicking on the node "Constructs and Concepts" in the center of this map causes KMap to send a message to Netscape requesting that the url
"http://ksi.cpsc.ucalgary.ca/PCP/PCPInto.html\#4" be fetched. This is a section of an HTML document that NetScape then displays. Clicking on other nodes in the concept map causes NetScape to navigate to different sections of this document.

KMap is currently implemented only for the Apple Macintosh and hence can act as a client helper only on Macintosh computers. We are currently working on ports to Windows and Motif which will make KMap helpers available on all major platforms. However, there will always be users who do not have, or want to use, the helpers but where it is appropriate to provide non-editable concept maps as clickable maps in HTML documents. We have interfaced KMap as an auxiliary server through the common gateway interface to MacHTTP to allow the same concept maps to be used as clickable maps.

Figure 6 shows the file fetched when the user clicks on the "access the same KSI material" in the second paragraph of the document of Figure 5. KMap converts the concept map to a GIF file and delivers it as an image that Netscape can read.

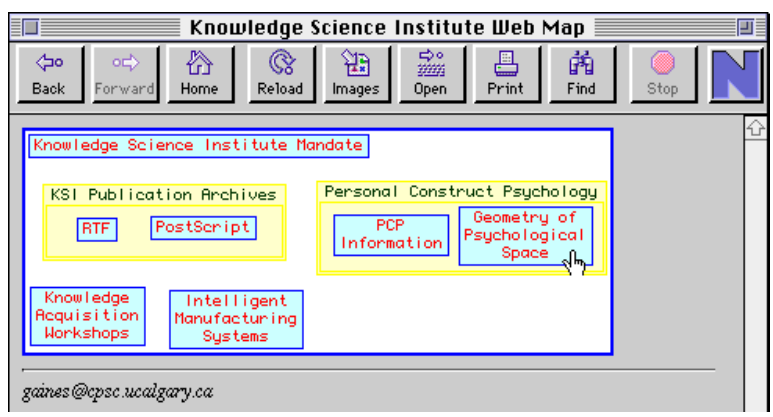

Figure 6 KMap acting as a server through the common gateway interface to MacHTTP

Clicking on the node "Geometry of Psychological Space" sends the coordinates of the point clicked back to KMap on the server which then takes the same action as if the map had been clicked in a helper. Thus KMap supports the use of concept maps on WorldWide Web through client helpers and server gateways in an integrated way.

\section{Applications to Collaborative Learning}

We have previously reported experience in the use of concept maps to support creative processes in collaborative learning situations (Gaines and Shaw, 1993). Our approach has been to have individual students develop concept maps for their domain of interest and link them to associated materials. Other students then critique these maps, modifying them, or adding to them, to provide alternative versions.

Sometimes consensual maps are developed by negotiation. At other times the absence of such consensus is the major learning experience since it demonstrates the plurality of incompatible perspectives available. 
Students working in collaborative groups usually divide responsibility for the domain, and produce networks of linked maps and associated materials. These can grow to become very complex, particularly if they are part of long-term activitie such as those of a research group.

One of the problems in the past has been to support concept mapping across distributed groups, and to make the materials developed more widely available. The move to World-Wide Web overcomes the limitations of tools operating only on local personal computers, and the capability to use concept maps as WWW "clickable maps" makes it possible to provide access to material to anyone using any platform with a WWW browser.

\section{Conclusions}

Concept maps have a long history of being used in support of learners and, in general form, to support a wide variety of visual thought processes in individuals and groups. This article has shown how computerbased concept mapping tools may be used to support collaborative learning by sharing maps on personal computers, working together on linked maps on different workstations, and sharing maps across WorldWide Web. In particular, it has shown that a single tool can be designed to have the flexibility to operate in all these modes. The class library architecture used to implement KMap is described in detail elsewhere (Gaines, 1994).

The graphic capabilities necessary to support interactive concept mapping are currently missing in WWW protocols and browsers. We see them as a major omission, and are committed to providing concept map support on WWW, through client helpers for the major platforms that will ultimately support maps embedded in documents through OLE II and OpenDoc protocols.

We believe that concept mapping techniques accessible through the World-Wide Web will provide significant support for many forms of collaborative learning.

\section{Acknowledgments}

This work was funded in part by the Natural Sciences and Engineering Research Council of Canada.

\section{References}

Arnheim, R. (1969). Visual Thinking. Berkeley, California, University of California Press.

Axelrod, R. (1976). Structure of Decision. Princeton, New Jersey, Princeton University Press.

Gaines, B.R. (1994). Class library implementation of an open architecture knowledge support system.
International Journal Human-Computer Studies 41(1/2) 59-107.

Gaines, B.R. and Shaw, M.L.G. (1993). Supporting the creativity cycle through visual languages. AAAI Spring Symposium: AI and Creativity. pp.155-162. Menlo Park, California, AAAI.

Gaines, B.R. and Shaw, M.L.G. (1994). Concept maps indexing multimedia knowledge bases. AAAI94 Workshop: Indexing and Reuse in Multimedia Systems. pp.36-45. Menlo Park, California, AAAI.

Glinert, I.P., Ed. (1990). Visual Programming Environments: Paradigms and Systems. Los Alamitos, California, IEEE Computer Society Press.

Lambiotte, J.G., Dansereau, D.F., Cross, D.R. and Reynolds, S.B. (1989). Multirelational semantic maps. Educational Psychology Review 1(4) 331-367.

Nersessian, N.J. (1989). Conceptual change in science and in science education. Synthese 80(1) 163184.

Nielson, G.M. and Rosenblum, L., Ed. (1991). Visualization'91: Proceedings of the Second IEEE Conference on Visualization. Los Alamitos, California, IEEE Computer Society Press.

Novak, J.D. (1977). A Theory of Education. Ithaca, Illinois, Cornell University Press.

Novak, J.D. and Gowin, D.B. (1984). Learning How To Learn. New York, Cambridge University Press.

Thadgard, P. (1992). Conceptual Revolutions. Princeton, New Jersey, Princeton University Press.

Toulmin, S. (1958). The Uses of Argument. Cambridge, UK, Cambridge University Press.

\section{Authors Addresses}

Brian $R$ Gaines and Mildred L G Shaw: Knowledge Science Institute, University of Calgary, Calgary, Alberta, Canada T2N 1N4. \{gaines, mildred @ cpsc.ucalgary.ca. 